\title{
Characteristic imaging finding and spot radiological diagnosis in a young man with acute breathlessness and chest pain
}

\author{
Mayank Mishra', Subodh Kumar² \\ 'All India Institute of Medical Sciences, Rishikesh, India \\ ${ }^{2}$ All India Institute of Medical Sciences, Gorakhpur, India
}

Septic pulmonary embolism (SPE) is a serious complication of fulminant bacteraemia, usually seen in immunocompromised persons or intravenous drug abusers. Prompt recognition is usually possible through classic imaging appearance in the relevant clinical background.

A 26-year-old, previously-healthy, immunocompetent male presented to the pulmonology outpatient centre with sudden onset of breathlessness, chest pain, dry cough and burning micturition of three days' duration. A week earlier he had consulted the orthopaedic outpatient centre for acute high-grade fever with a painfully swollen left knee, and was diagnosed as left distal femur acute osteomyelitis. Immediately prior to this presentation, he had developed a furuncle in the left external nostril that went unattended. There was no history of trauma, intravenous drug abuse or other addictions. Examination revealed a temperature of $101 \mathrm{~F}$, blood pressure $126 / 82 \mathrm{~mm} \mathrm{Hg}$, pulse rate $124 / \mathrm{min}$, respiratory rate $28 / \mathrm{min}$ and $\mathrm{SPO}_{2} 89 \%$ on ambient air. The left knee joint was swollen and tender. Investigations were remarkable for a total leukocyte count of 29,100/ $\mathrm{mm}^{3}$ and C-reactive protein $151 \mathrm{mg} / \mathrm{dL}$. Blood and urine cultures were positive for methicillin-resistant Staphylococcus aureus (MRSA), while sputum and synovial fluid cultures were sterile. Echocardiography was unremarkable, and chest radiograph showed bilateral ill-defined nodules (Figure 1A). Computed tomogram of the chest (Figure 1B) revealed multiple, bilateral, variably-sized, pleural-based and parenchymal nodules, few of them with cavitation. Some of the nodules had a distinct vessel branch directly entering it, suggestive of the classical feeding vessel sign (FVS).

The clinical picture and pathognomonic imaging features suggested a diagnosis of SPE secondary to left distal femur acute osteomyelitis-induced MRSA septicaemia. The patient was initiated on linezolid and cefepime intravenously as per the culture-sensitivity reports, which was followed by respiratory stabilization within 72 hours and complete clearing of pulmonary nodules after two weeks. He was subsequently transferred to orthopaedics for further care of his joint complaints.

SPE is an uncommon, potentially fatal complication of fulminant bacteraemia. It is usually seen in patients with right-heart infective endocarditis, indwelling devices and catheters, skin or soft tissue or bone infections, septic abortions, oro-dental infections and in immunocompromised persons. The occurrence of septic embolism in an immunocompetent host indicates severe infection that fails to remain contained at the primary focus and spreads to other sites. SPE arises due to hematogenous dissemination of infected microthrombi from an extrathoracic nidus. The thromboemboli produce microvascular occlusion within the lungs resulting in a host of insults that include ischaemic lung parenchymal damage, infarction, infective and inflammatory changes, and microabscess formation. These pathophysiological processes manifest as lung nodules on chest imaging.

A high clinico-radiologic index of suspicion is required as the diagnosis of SPE can be challenging due to diverse presentations and varying aetiologies. The most commonly reported clinical features include chest pain, dyspnoea and cough occurring in a febrile patient with a known extrathoracic site of bacteraemia. Blood cultures, chest imaging and echocardiography form the cornerstone of diagnosis of suspected SPE. Diagnosis relies greatly on the classical chest CT findings in an appropriate clinical setting. Typical CT imaging characteristics include bilateral, peripheral nodules (with or without cavitation), wedge-shaped pleural-based infarcts, FVS, pleural effusion, and mediastinal and/or hilar lymphadenopathy [1-3]. FVS is considered a highly suggestive imaging sign of SPE, with a reported prevalence of $67-100 \%$ [4]. It is also known as the feeding artery sign or fruits on the branch sign, and indicates a pulmonary artery branch that leads directly to the nodule. Other conditions where it may be commonly seen are lung metastases, pulmonary infarcts, vasculitis, and pulmonary arteriovenous malformations [5].

Management of SPE involves adequately addressing the primary focus of infection, prolonged course of broad-spectrum antibiotics, and other supportive measures as appropriate. Complications depend upon the

Address for correspondence: Mayank Mishra, All India Institute of Medical Sciences, Rishikesh, India; e-mail: virgodrmayank@gmail.com Conflict of interest: None declared

DOI: 10.5603/ARM.a2021.0073 | Received: 10.02.2021 | Copyright (C) 2021 PTChP | ISSN 2451-4934 | e-ISSN 2543-6031

This article is available in open access under Creative Common Attribution-Non-Commercial-No Derivatives 4.0 International (CC BY-NC-ND 4.0) license, allowing to download articles and share them with others as long as they credit the authors and the publisher, but without permission to change them in any way or use them commercially. 

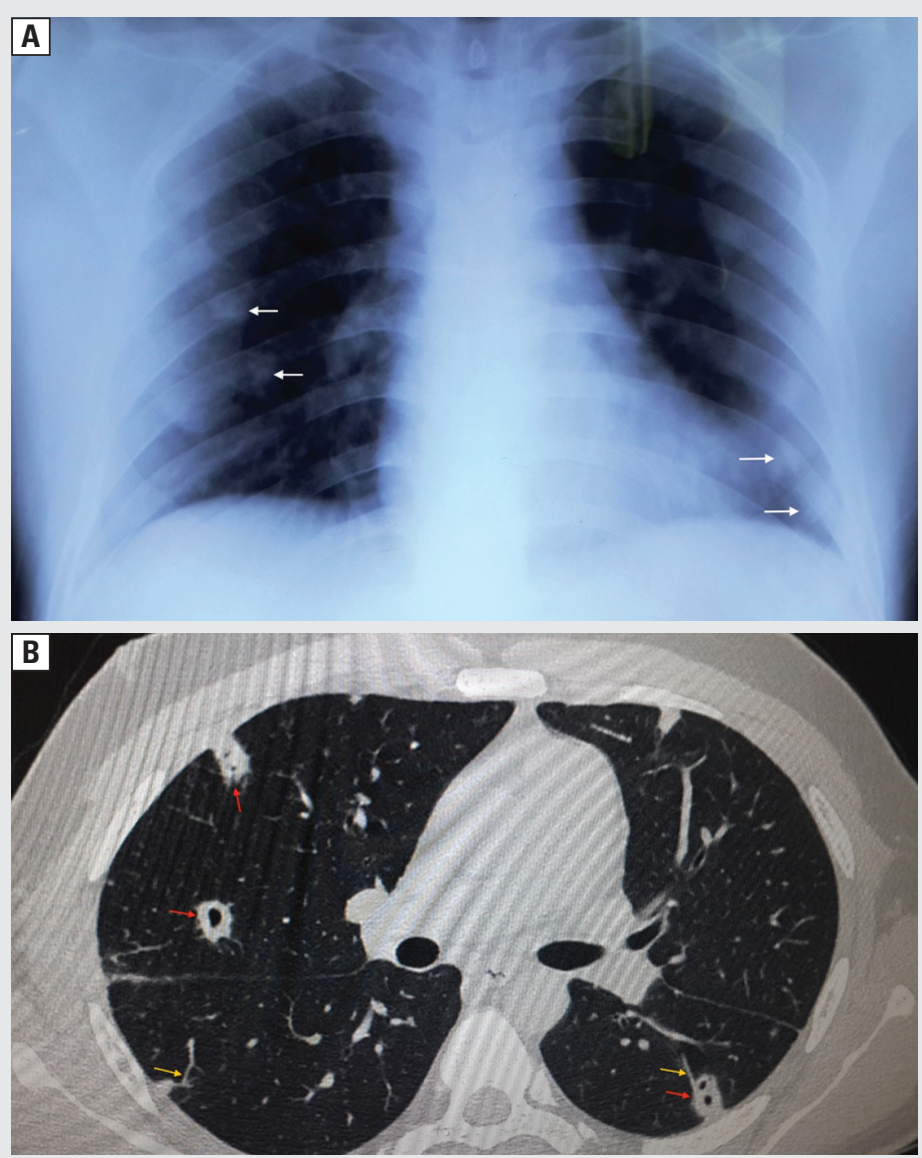

Figure 1. A. Chest radiograph (posteroanterior view) showing bilateral, ill-defined, nodular parenchymal opacities, mainly in mid and lower lung zones (white arrows). B. Chest computed tomogram scan (axial section, lung window) showing multiple, bilateral, peripheral parenchymal nodules, few showing cavitation (red arrows), and some having a distinct vessel branch directly entering the nodule — the feeding vessel sign (yellow arrows)

quantum of lung parenchymal damage inflicted, and include empyema, lung abscess, chronic cavitary lung disease, pneumothorax, and acute hypoxemic respiratory failure.

To conclude, SPE is a serious condition that must be strongly considered in the appropriate clinical context. Thoracic CT imaging plays an invaluable role in diagnosis especially when characteristic imaging findings are present. Timely diagnosis and aggressive treatment of this entity can be life-saving. This clinical vignette is being reported to highlight the pathognomonic FVS that facilitates clinching of the diagnosis of this uncommon entity, and must incite its consideration even in young immunocompetent individuals without any comorbidities or risk factors.

\section{References:}

1. Kuhlman JE, Fishman EK, Teigen C. Pulmonary septic emboli: diagnosis with CT. Radiology. 1990; 174(1): 211-213, doi: 10.1148/ radiology.174.1.2294550, indexed in Pubmed: 2294550.

2. Cook RJ, Ashton RW, Aughenbaugh GL, et al. Septic pulmonary embolism: presenting features and clinical course of 14 patients. Chest. 2005; 128(1): 162-166, doi: 10.1378/chest.128.1.162, indexed in Pubmed: 16002930.

3. Wong KS, Lin TY, Huang YC, et al. Clinical and radiographic spectrum of septic pulmonary embolism. Arch Dis Child. 2002; 87(4): 312-315, doi: 10.1136/adc.87.4.312, indexed in Pubmed: 12244005.

4. Dodd JD, Souza CA, Müller NL. High-resolution MDCT of pulmonary septic embolism: evaluation of the feeding vessel sign. AJR Am J Roentgenol. 2006; 187(3): 623-629, doi: 10.2214/AJR.05.0681, indexed in Pubmed: 16928922.

5. Yudin A. Feeding Vessel Sign or Fruits on the Branch Sign. In: Metaphorical Signs in Computed Tomography of Chest and Abdomen. Springer, Cham https://doi org/10 1007/978-3-319-04013-4, Cham 2014: 22-25. 